REVIEW ARTICLE

\title{
Hypothalamic primary cilium: A hub for metabolic homeostasis
}

\author{
Dong Joo Yang ${ }^{1}$, Jessica Hong ${ }^{2}$ and Ki Woo Kim (DD ${ }^{1 凶}$
}

(c) The Author(s) 2021

Obesity is a global health problem that is associated with adverse consequences such as the development of metabolic disorders, including cardiovascular disease, neurodegenerative disorders, and type 2 diabetes. A major cause of obesity is metabolic imbalance, which results from insufficient physical activity and excess energy intake. Understanding the pathogenesis of obesity, as well as other metabolic disorders, is important in the development of methods for prevention and therapy. The coordination of energy balance takes place in the hypothalamus, a major brain region that maintains body homeostasis. The primary cilium is an organelle that has recently received attention because of its role in controlling energy balance in the hypothalamus. Defects in proteins required for ciliary function and formation, both in humans and in mice, have been shown to cause various metabolic disorders. In this review, we provide an overview of the critical functions of primary cilia, particularly in hypothalamic areas, and briefly summarize the studies on the primary roles of cilia in specific neurons relating to metabolic homeostasis.

Experimental \& Molecular Medicine (2021) 53:1109-1115; https://doi.org/10.1038/s12276-021-00644-5

\section{INTRODUCTION}

The cilium is a hair-like organelle formed with cell membrane and is present on nearly every mammalian cell. Cilia have historically been classified as either motile or immotile. Structurally, a cilium consists of a microtubule-based axoneme covered by a ciliary membrane (Fig. 1). The axoneme emerges from the basal body, a centriole-derived and microtubule-organizing center, extending from the cell surface into the extracellular space [1]. In terms of their formation and functions, cilia are constructed through a microtubule motor-based transport system that consists of intraflagellar transport (IFT) complexes that bind directly to cargos and their motors kinesin-2 and dynein, enabling travel across the axoneme [2, 3]. The IFT complex consists of two distinct subcomplexes, complexes $A$ and $B$. Complex $A$ is needed for retrograde movement from the ciliary tip to the cytoplasm, while complex $B$ is utilized in anterograde transport from the cellular base to the ciliary tip. Balanced transport systems are important for ciliogenesis, as faulty regulation of these factors causes abnormal cilia formation [3].

Most mammalian cells have a single immotile cilium, called the primary cilium, which has evolved to receive various signals from extracellular stimuli $[1,4]$. Due to their ability to detect sensory cues, primary cilia are considered cellular antennae. However, little attention had been directed to the importance of primary cilia until the discovery of their association with polycystic kidney disease (PKD) [5]. PKD is an inherited genetic disorder that results in progressive renal cyst formation due to abnormal primary cilia function. Later, the medical significance of primary cilia, beyond their relation to PKD, became increasingly evident, with evidence showing that the structural and functional anomalies of primary cilia arise from genetic mutations in ciliary proteins. These proteins are closely related to human diseases comprehensively called ciliopathies, including retinal degeneration, polydactyly, hypertension, and obesity [6]. While it has been established that primary cilia have critical and diverse functions in several cell types, many questions regarding the precise functions of primary cilia in these cell types, particularly neuronal primary cilia, remain.

Body weight regulation has garnered significant attention due to its importance in maintaining energy balance, which is primarily determined by food intake and energy expenditure. Research on feeding behavior and energy consumption is needed to cure metabolic diseases such as obesity and diabetes, along with their complications. The brain, particularly the hypothalamus, plays a critical role in integrating and coordinating several types of signals, including hormones and nutrients, to maintain body homeostasis. Recently, primary cilia have received attention because of their functioning as sensory centers in controlling energy balance. Early evidence linking primary cilia and energy homeostasis was realized upon the discovery that proteins associated with human obesity syndromes, such as Alström and Bardet-Biedl syndromes, localize to primary cilia. Mutations in these proteins, both in humans and in mice, result in organisms displaying severe obesity and diabetes [6-8]. In 2007, Davenport et al. directly assessed the importance of primary cilia by utilizing conditional knockout (KO) of ciliogenesis genes ( $T g 737$ and Kif3a). This study was the first to show that neuronal primary cilia are required for normal energy homeostasis [9]. Since then, neuronal primary cilia have emerged as critical organelles in the integration of the complex signals in metabolic homeostasis [10-12]. Therefore, in this review, we summarize the evidence supporting the role of hypothalamic primary cilia in controlling metabolic homeostasis.

\section{MAIN TEXT}

The hypothalamus, a site for energy balance control

The hypothalamus is a key brain region in the balance of body homeostasis [13, 14]. It encompasses several anatomically

\footnotetext{
${ }^{1}$ Departments of Oral Biology and Applied Biological Science, BK21 Four, Yonsei University College of Dentistry, Seoul 03722, Korea. ${ }^{2}$ Brown University, Providence, RI 02912, USA. 凶email: KIWOO-KIM@yuhs.ac
} 

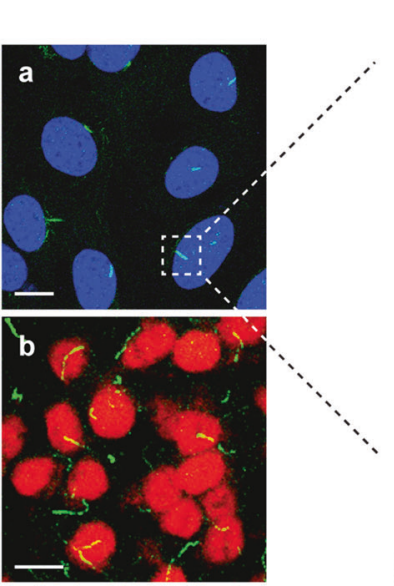

c
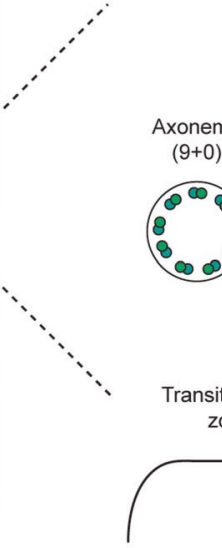

\section{2}

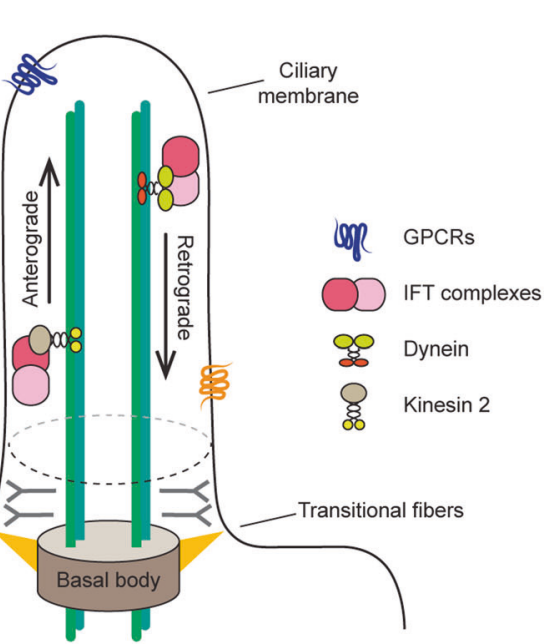

Fig. 1 Schematic structure of primary cilia. Immunofluorescence images of primary cilia (green, ADCY3) in hypothalamic cells (a) and arcuate nuclei (b). Scale $=20 \mu \mathrm{m}$. Schematic structure of primary cilia (c). The primary cilium is an antenna-like organelle that receives diverse signals from the extracellular environment. It is comprised of the ciliary membrane surrounding the microtubule-based axoneme. The nine parallel microtubule doublets of the axoneme, which show " $9+0$ " rings, form the backbone of the appendage, while the basal body acts as a microtubule-organizing center. The components that are transported from the basal body to the ciliary tip by anterograde transport rely on the intraflagellar transport (IFT) protein attached to the motor protein kinesin 2. In contrast, retrograde transport from the ciliary tip to the cytoplasm depends on dynein motor proteins. The ciliary membrane is highly enriched with several receptors, including $\mathrm{G}$ protein-coupled receptors (GPCRs).

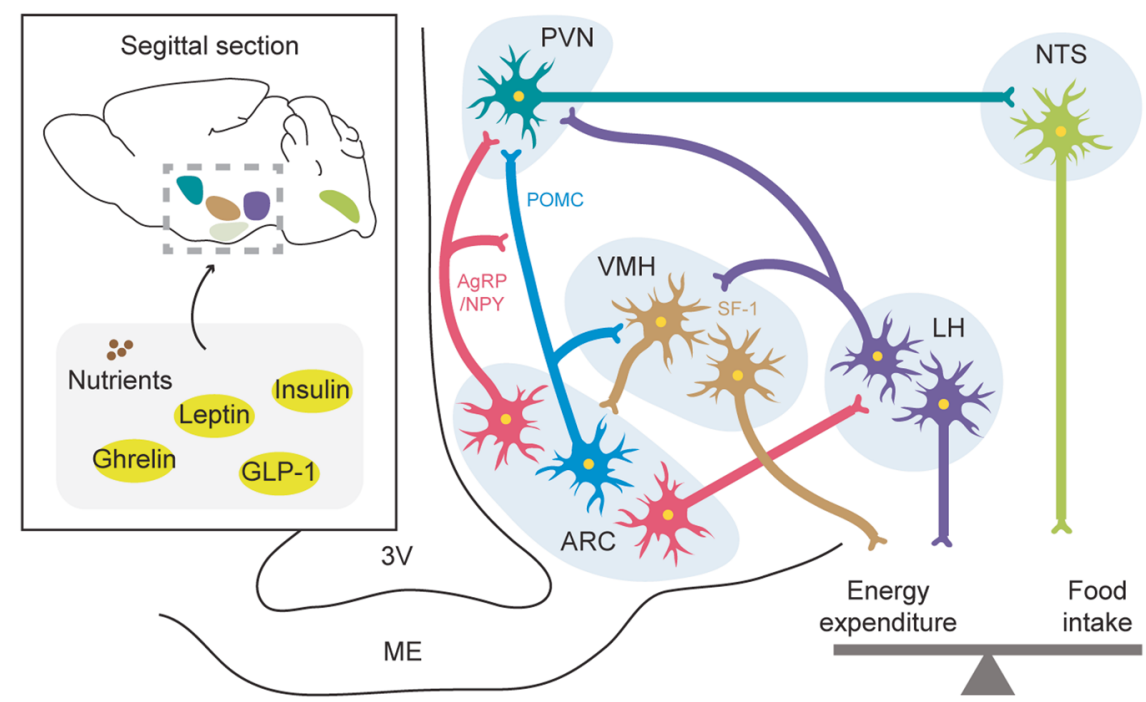

Fig. 2 Hypothalamic nuclei involved in the regulation of energy balance. Energy homeostasis is regulated by a complex feedback loop involving endocrine and neuronal signals originating from peripheral organs and intrahypothalamic communications. The ARC is a key nucleus that houses POMC and AgRP/NPY neurons, which integrate the aforementioned signals. These neurons project to various nuclei, including the PVN, VMH, and LH. In turn, the ARC receives input from the VMH and LH. The NTS receives projections from the ARC, PVN, VMH, and $\mathrm{LH}$ and regulates multiple metabolic effectors of energy balance. $3 \mathrm{~V}$, third ventricle; AgRP, agouti-related protein; ARC, arcuate nucleus; $\mathrm{LH}$, lateral hypothalamus; ME, median eminence; NPY, neuropeptide Y; NTS, nucleus of tractus solitarius; POMC, proopiomelanocortin; PVN, paraventricular nucleus; SF-1, steroidogenic factor 1; and VMH, ventromedial hypothalamus.

well-defined nuclei, including the arcuate nucleus (ARC), as well as the ventromedial hypothalamus (VMH), dorsomedial hypothalamus (DMH), lateral hypothalamus (LH), and paraventricular nucleus (PVN) of the hypothalamus (Fig. 2) [15]. The ARC, which is located at the base of the hypothalamus and is in close proximity to the median eminence (ME), primarily senses metabolic signals from the periphery via systemic circulation. There are two distinct functionally antagonistic neurons: (i) orexigenic agouti-related peptide (AgRP)- and neuropeptide $\mathrm{Y}$ (NPY)-expressing neurons and (ii) anorexigenic proopiomelanocortin (POMC)-expressing neurons $[16,17]$. These neuronal populations are called first-order neurons because they integrate peripheral, nutritional, and hormonal cues to control energy balance $[18,19]$ through various hormone receptors distributed over the neuronal membrane, such as leptin receptor (LepR) and insulin receptor (IR) [20]. For example, leptin binds to receptors expressed at the surface of POMC and AgRP neurons. Once leptin binds to LepR, the neurons are either activated or inhibited and regulate food intake and energy expenditure by releasing melanocortin peptides, which are key products that control energy balance. POMC neurons produce a-melanocyte-stimulating hormone (a-MSH), an agonist of melanocortin receptor 4 (MC4R), which is key in regulating energy and glucose homeostasis. In contrast, the inverse agonist AgRP suppresses MC4R 
activity and simultaneously antagonizes the effects of a-MSH $[18,21,22]$. These neurons project to both intrahypothalamic neurons (e.g., neurons in the $\mathrm{VMH}, \mathrm{DMH}, \mathrm{LH}$, and PVN) and extrahypothalamic neurons (e.g., the nucleus of tractus solitarius (NTS) and the mesolimbic reward system), communicating information regarding peripheral energy availability to other brain areas (Fig. 2) [23]. Among these areas, the PVN seems to be the center of the melanocortin system. Once either AgRP or a-MSH binds to MC4R in the neurons of the PVN, feeding behavior is modulated, possibly through the autonomic nervous system [24-29].

The PVN lies alongside the top portion of the third ventricle (3 V) in the anterior hypothalamus, and it plays an imperative role in the regulation of energy balance and endocrinological activities. Lesions in the PVN produce obesity and hyperphagia via control of the sympathetic outflow to peripheral metabolic organs [30, 31]. PVN neurons also constitute the primary endocrine control center, synthesizing and secreting neuropeptides that have a net catabolic action, such as corticotropin-releasing hormone (CRH) [32] and thyrotropin-releasing hormone (TRH) [33].

The VMH is an oval-shaped hypothalamic nucleus located directly above the ARC. This brain area contains neurons that sense glucose and leptin and thus is the site controlling the regulation of body weight and glucose homeostasis [34, 35]. A number of studies have shown that steroidogenic factor-1 (SF-1) neurons in the $\mathrm{VMH}$ actively participate in insulin and leptin signaling in energy expenditure and glucose homeostasis, exerting minor effects on feeding behavior [36-38]. In addition, VMH neurons can modulate sympathetic nerve activity (SNA), which is thought to underlie a variety of neuronal mechanisms in the $\mathrm{VMH}[37,39-43]$. For instance, electrical stimulation of the $\mathrm{VMH}$ induces glycogenolysis and gluconeogenesis in the liver and increases blood pressure and heart rate through sympathoexcitation $[44,45]$, whereas destruction of the $\mathrm{VMH}$ by bilateral lesions causes hyperphagia, obesity, hyperglycemia, and reduced SNA $[14,46,47]$. POMC neurons in the ARC project to the $\mathrm{VMH}$ and control food intake via stimulation of MC4R and subsequent activation of brain-derived neurotrophic factor (BDNF) [48]. On the other hand, the $\mathrm{VMH}$, which consists mostly of glutamatergic neurons, projects to the ARC and transduces excitatory input to both POMC and AgRP neurons [49].

The $\mathrm{LH}$ is important in receiving sensory signals from the periphery, including the gut and liver $[50,51]$. This brain region contains large numbers of glucose-receptive neurons that respond to circulating glucose levels, most likely via pathways ascending from the hypothalamus [52]. In contrast to the PVN and $\mathrm{VMH}$, bilateral destruction of the lateral portion of the $\mathrm{LH}$ abolishes food intake, thus resulting in weight loss in rats, even in those who had previously been induced to obesity [14]. Therefore, the LH is considered a feeding center in the hypothalamus.

Overall, the diverse neuronal networks of various hypothalamic regions are instrumental in regulating energy balance, each individually functioning to maintain body homeostasis.

\section{Neuronal primary cilia and regulation of body homeostasis}

While it has been known for decades that primary cilia exist in neurons, their precise functions in each type of neuron remain poorly understood. In the past 20 years, the importance of neuronal cilia has been reported through various studies, and the evidence for this importance is increasing daily. An early observation showing a correlation between metabolic disorders and neuronal primary cilia function was based on conditional tamoxifen-inducible KO of the core ciliogenesis gene Kif3a and the intraflagellar transport 88 (Ift88, also called Tg737) protein [9]. Germline ablation of either Kif3a or Tg737 in adult mice resulted in hyperphagia-induced obesity with elevated serum leptin, insulin, and glucose levels. Restricting dietary intake prevented increases in both body weight and serum hormones, supporting the notion that the obesity and diabetic phenotypes were a consequence of hyperphagic behaviors. Interestingly, specific deletion of Kif3a or Tg737 using synapsin 1-cre (Syn1-cre) led to similar results of obesity and leptin resistance, indicating that neuronal primary cilia may play important roles in the regulation of body weight homeostasis [9].

Adenylyl cyclase 3 (ADCY3) catalyzes the synthesis of cyclic AMP (CAMP), an important second messenger in signaling pathways, from ATP $[53,54]$. In the central nervous system (CNS), CAMP plays a critical role in neuronal functions, including survival, growth, differentiation, and synaptogenesis [55]. In 2007, neuronal cilia were found to be specifically enriched with ADCY3 [56], and it has subsequently been shown that global Adcy3-KO mice exhibit adult onset obesity due to disruption of cAMP signaling in the hypothalamus [57]. In addition, a number of $G$ protein-coupled receptors (GPCRs), including somatostatin receptor 3 (SSTR3), serotonin receptor 6 (5-HT6R), melanin-concentrating hormone receptor 1 (MCHR1), and more recently MC4R, have been reported to be located in neuronal cilia [58]. Once ciliary components are defective, aberrant ciliary localization and signaling of certain GPCRs are displayed, causing obesity.

Overall, these results indicate that both the structural and functional roles of neuronal primary cilia are necessary to control energy homeostasis. Indeed, several recent pieces of evidence suggest that hypothalamic cilia may function together as a metabolic signaling center, which is critical to the control of body homeostasis (Table 1) [9, 41, 59-62].

\section{Primary cilia in the arcuate nucleus}

Primary cilia in both orexigenic and anorexigenic neurons in the ARC have been implicated not only in the regulation of food intake but also in responses to hormones, including leptin and insulin. Systemic ablation of ciliary genes from neurons using Syn1-cre led to hyperphagic-induced obesity, and the obesity phenotype of both Kif3a-Syn1-KO and Tg737-Syn1-KO mice was reproduced in POMC-specific Kif3a-KO (Kif3a-Pomc-KO) mice [9]. Both male and female Kif3a-Pomc-KO mice exhibited significant increases in weight, primarily due to hyperphagia. Deletion of Kif3a in POMC neurons also led to elevated levels of leptin and insulin, indicating that cilia in POMC neurons are required to maintain both energy balance and responses to satiety signals such as leptin and insulin signals. Furthermore, introduction of short inhibitory RNA (siRNA) targeting Kif3a or Ift88 in the ARC led to an increase in food intake and a decrease in energy expenditure, manifesting the obese phenotype [63].

A recent study showed that inhibition of ciliogenesis in developing POMC neurons, which was realized by depleting Kif3a or Ift88, led to adulthood obesity in mice [64]; these mice showed disruption of axonal projections through impaired lysosomal protein degradation in POMC neurons. In contrast, ciliary deletion in adult POMC neurons using tamoxifen-inducible cre did not lead to significant changes in body weight, fat mass, or lean mass, suggesting that primary cilia in adult POMC neurons have a minimal role in the regulation of energy balance.

Bardet-Biedl syndrome (BBS) is a rare recessive genetic disease, and its patients commonly display severe obesity $[7,8]$. The BBS protein complex, a subset of the IFT complex, also participates in the transportation of ciliary membrane proteins $[65,66]$. In contrast to Ift88 and Kif3a, loss-of-function mutations in BBS genes do not lead to complete structural defects of primary cilia; however, they disrupt ciliary functions [67]. Germline ablation of

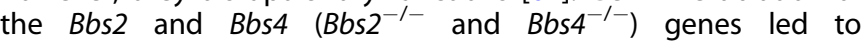
hyperphagia-induced obesity, coupled with reduced phosphorSTAT3 levels in the hypothalamus. Furthermore, research has shown that a lack of localization of SSTR3 and MCHR1 to cilia in $B b s 2^{-1-}$ and $B b s 4^{-1-}$ mouse neurons [68], indicating that the altered signaling caused by mislocalization of ciliary signaling 
Table 1. Metabolic phenotypes of hypothalamic primary cilia dysfunction.

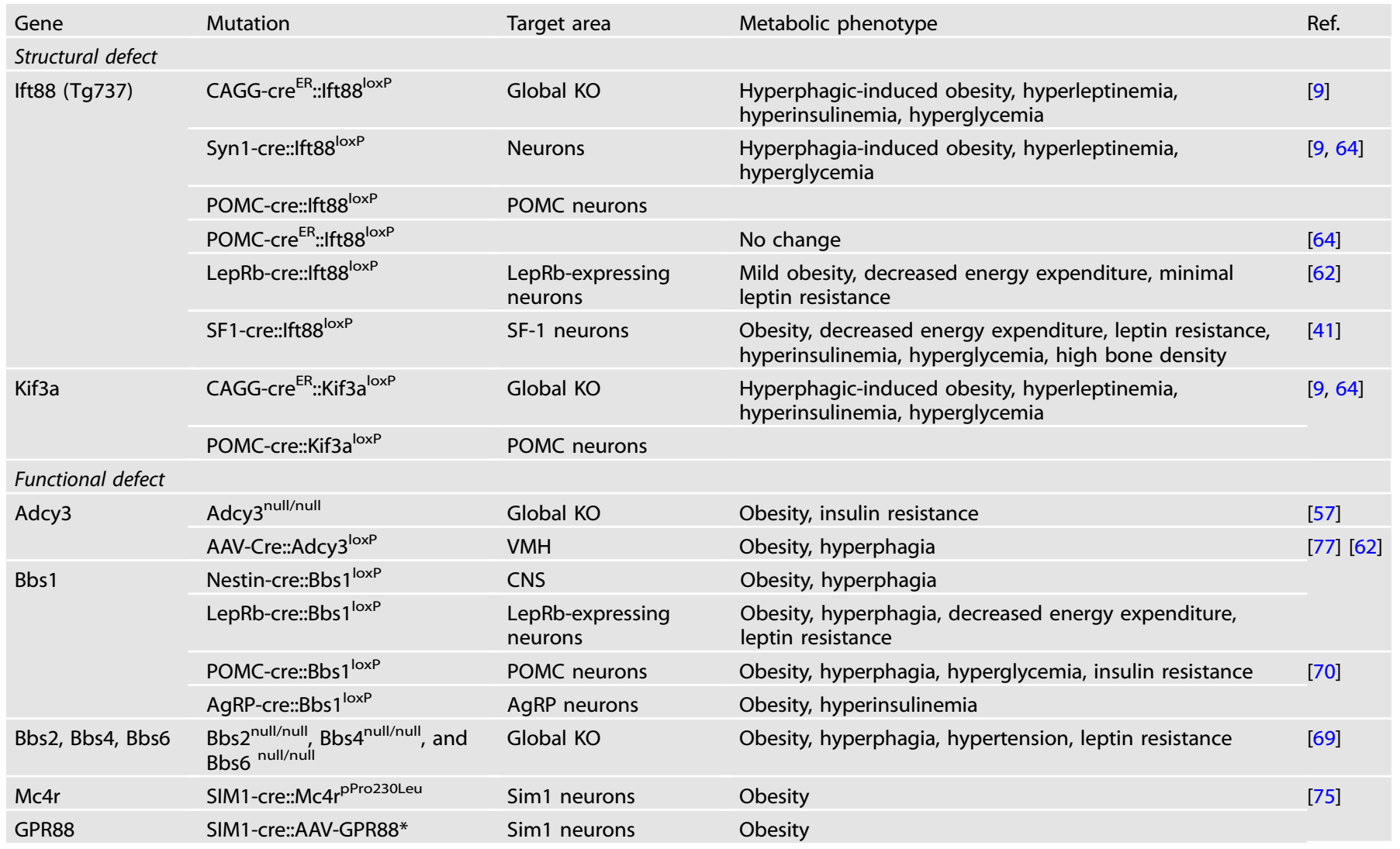

${ }^{*} \mathrm{G}$ protein-coupled receptor 88

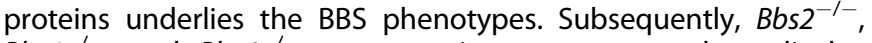
$B b s 4^{-1-}$, and $B b s 6^{-1-}$ mutant mice were reported to display hyperleptinemia as a result of defective leptin signaling [61, 69]. Molecular studies revealed that Bbs1, a component of the BBSome, directly bound to the leptin receptor long-form (LepRb) and participated in LepRb trafficking. Consistently, Bbs1 M390R, which is the most common mutant of Bbs1 found in patients, showed decreased interaction with LepRb, implying that the LepRb trafficking and subsequent signaling pathways may be altered in BBS mutant patients. These studies suggest that ciliary BBS proteins might be required for normal leptin signaling and thus for the maintenance of energy balance [61]. Later, the results of studies on targeted disruption of the BBSome by deletion of the Bbs 1 gene in the brain using Nestin-cre (Nestin ${ }^{C r e} /$ Bbs $^{1 / f / 1}$ ) showed obesity in mice, and the obese phenotype was reproduced by ablation of Bbs1 in LepRb-expressing neurons, $\mathrm{LRb}^{\mathrm{Cre}} / \mathrm{Bbs}_{1} 1^{\mathrm{f} / \mathrm{fl} 62}$. Both Nestin ${ }^{C r e} / \mathrm{Bbs}^{\mathrm{f} / \mathrm{fl}}$ and $\mathrm{LRb}^{\mathrm{Cre}} / \mathrm{Bbs}^{\mathrm{f} / \mathrm{fl}}$ mice demonstrated increased food intake coupled with reduced hypothalamic Pomc levels. On the other hand, disruption of Ift88 in LepRb-expressing cells caused minimal gains in body weight and fat mass. In contrast to $\mathrm{LRb} b^{\mathrm{Cre}} / \mathrm{Bbs} 1^{\mathrm{fl} / \mathrm{fl}}$, no difference in food intake was found in $\mathrm{LRb}^{\mathrm{Cre}} / \mathrm{Ift} 88^{\mathrm{f} / \mathrm{fl}}$ mice; however, this model showed a decrease in both energy expenditure and body temperature. Moreover, mechanistic studies demonstrated that the deletion of the expression of BBS proteins, but not Ift88, impaired LepRb trafficking to the plasma membrane, leading to central leptin resistance in a manner independent of obesity. In summary, these results demonstrated that cilium-mutant mouse models may display obesity through different and independent mechanisms.

Recent studies from the same group that performed the LepRb trafficking study reported the role of Bbs1 in POMC and AgRP neurons. Deletion of Bbs1 in either POMC or AgRP neurons led to result similar to those obtained with models of leptin-signaling deficiency [70]. Both POMC ${ }^{\mathrm{Cre}} / \mathrm{Bbs}^{\mathrm{fl} / \mathrm{fl}}$ and $\mathrm{AgRP} \mathrm{Cre}^{\mathrm{Cr}} / \mathrm{Bbs} 1^{\mathrm{f} / \mathrm{fl}}$ mice showed obesity associated with an increase in food intake. In addition, by lacking the BBSome protein, as identified by the impairment of serotonin receptor $5-\mathrm{HT}_{2} \mathrm{R}$, it was shown that trafficking to the membrane contributed to the hypothalamic BBSome control of energy balance and handling of metabolic receptors [70].

\section{Abnormal functions of primary cilia in the paraventricular nucleus cause obesity}

MC4R is a central component of the melanocortin system, a hypothalamic network that integrates information from the periphery and regulates food intake and energy expenditure [71]. Mutations in $\mathrm{Mc} 4 \mathrm{r}$ are the most common monogenic cause of severe obesity in humans, as well as in rodents [72-74]. Although the expression pattern of MC4R has been well documented, previous attempts to determine the subcellular localization of MC4R in vivo have been unsuccessful. In 2018, it was first reported that MC4R localizes to primary cilia in a subset of mouse hypothalamic neurons, including the PVN, where it colocalized with ADCY3 [75]. This finding was particularly relevant since it was previously reported that $A D C Y 3$ mutations are closely associated with human obesity, and Adcy3-KO mice exhibited obesity upon disruption of CAMP signaling in the hypothalamus [57]. In this study, it was discovered that the impaired localization of MC4R in primary cilia caused MC4R mutations, which led to suppression of ADCY3 activity in cilia and, in turn, the acquisition of the obese phenotype in mice [75]. Furthermore, specific inhibition of ADCY3 through the use of GPR88, a constitutively active version of the 
cilia-specific $\mathrm{Ga}_{\mathrm{i}}$-protein-coupled receptor, resulted in suppression of cAMP production and, consequently, increased body weight. These findings suggest that MC4R and ADCY3 may positively regulate CAMP generation in neuronal primary cilia of the PVN, where MC4R is highly expressed, and impaired cAMP signaling in the primary cilia of MC4R-expressing neurons leads to obesity. However, additional studies investigating whether primary cilia are required for $\mathrm{Ga}_{\mathrm{S}}$ coupling and ADCY3 activation by MC4R may be necessary. Moreover, since the primary cilia of MC4R-positive neurons are not solely limited to the PVN, it is necessary to observe the functions of cilia in other MC4R-positive neurons.

\section{The importance of primary cilia in the ventromedial hypothalamus}

It has been reported that Adcy3 gene polymorphisms are associated with obesity and are known to be exclusively expressed in neuronal cilia $[57,76]$. Selective ablation of Adcy3 by injection of AAV-Cre into the $\mathrm{VMH}$ of Adcy3-floxed mice significantly increased body fat and led to obesity, supporting the idea that $A d c y 3$ in the VMH plays an important role in the regulation of energy balance [77].

Recently, we addressed the homeostatic roles of $\mathrm{VMH}$-expressing primary cilia in our research. We deleted VMH-primary cilia by targeting the Ift88 gene using either steroidogenic factor 1 cre (SF1-Cre) or bilateral AAV-Cre injection and then monitored the metabolic changes [41]. The VMH-specific primary cilia KO (IFT88$\mathrm{KO}^{\mathrm{SF}-1}$ ) mice exhibited metabolic dysregulation linked to decreased sympathetic nervous activity (SNA) and central leptin resistance, which led to marked obesity. The obese phenotype of the IFT88 $\mathrm{KO}^{\mathrm{SF}-1}$ mice presented with decreased energy expenditure, which appeared to be a primary consequence of reduced sympathetic outflow rather than a secondary effect of obesity. In addition to the energy balance disturbance in the IFT88-KO $\mathrm{KF}^{\mathrm{SF}}$ mice, VMH-primary cilia have also been shown to be associated with bone density maintenance, suggesting that the altered sympathetic activity induced by deleting VMH-primary cilia might be critical for changing bone density [41]. Further studies delineating how VMH-primary cilia control SNA activity are necessary.

\section{Leptin signaling and primary cilia in the hypothalamus}

The most severe obesity phenotype in humans and mice results from a deficiency of either the leptin or the leptin receptor. Leptin deficiency-associated obesity is resolved upon treatment with recombinant leptin, strongly indicating the critical physiological roles of leptin and leptin signaling in the control of body energy homeostasis [78, 79]. One of the distinct phenotypes of ciliopathy is elevated leptin levels, indicating that leptin resistance may either be a cause or be a consequence of obesity [ 80,81$]$. Thus, the relationship between leptin action and the functional involvement of primary cilia has been investigated.

A potential molecular mechanism of cilia in leptin action was suggested by scientists studying BBS-mutant mice [61]. BBSmutant mice exhibited hyperphagia and higher leptin levels. Interestingly, they did not respond properly to leptin even after leptin levels were normalized by caloric restriction. Considering these results, Seo et al. suggested that the leptin-resistant phenotype in BBS-mutant mice may be a primary effect of cilia dysfunction [61]. However, Berbari et al. analyzed the leptin response in pre-obese, obese, and food-restricted mice after generating inducible Ift 88 and Bbs4 KOs [82] and found that the mutant mice showed leptin resistance only under obesityinducing conditions, strongly indicating that leptin resistance is a secondary consequence of obesity.

In another study, Guo et al. tested leptin sensitivity in $\mathrm{LRb}^{\mathrm{Cre}}$ / $B b s 1^{\mathrm{f} / \mathrm{fl}}$ mice that were at comparable body weight with WT littermates, but the response was substantially attenuated [62]. In addition, the $\mathrm{LRb}^{\mathrm{Cre}} / \mathrm{Bbs}^{\mathrm{fl} / \mathrm{fl}}$ mice under calorie-restricted conditions remained at a higher body weight and fat mass with lower energy expenditure, possibly due to reduction in leptin sensitivity and impaired leptin signaling. In addition to these experiments, the deletion of Ift88 in SF-1 neurons of the VMH also indicated the involvement of primary cilia in leptin action [41]. We measured leptin levels when the body weights of WT and IFT88-KO ${ }^{\mathrm{SF}-1}$ mice were comparable, simultaneously and directly injecting leptin and examining the leptin sensitivity in the WT and IFT88-KO ${ }^{\mathrm{SF}-1}$ mice. The physiological response to leptin in the IFT88-KO ${ }^{\mathrm{SF}-1}$ mice,

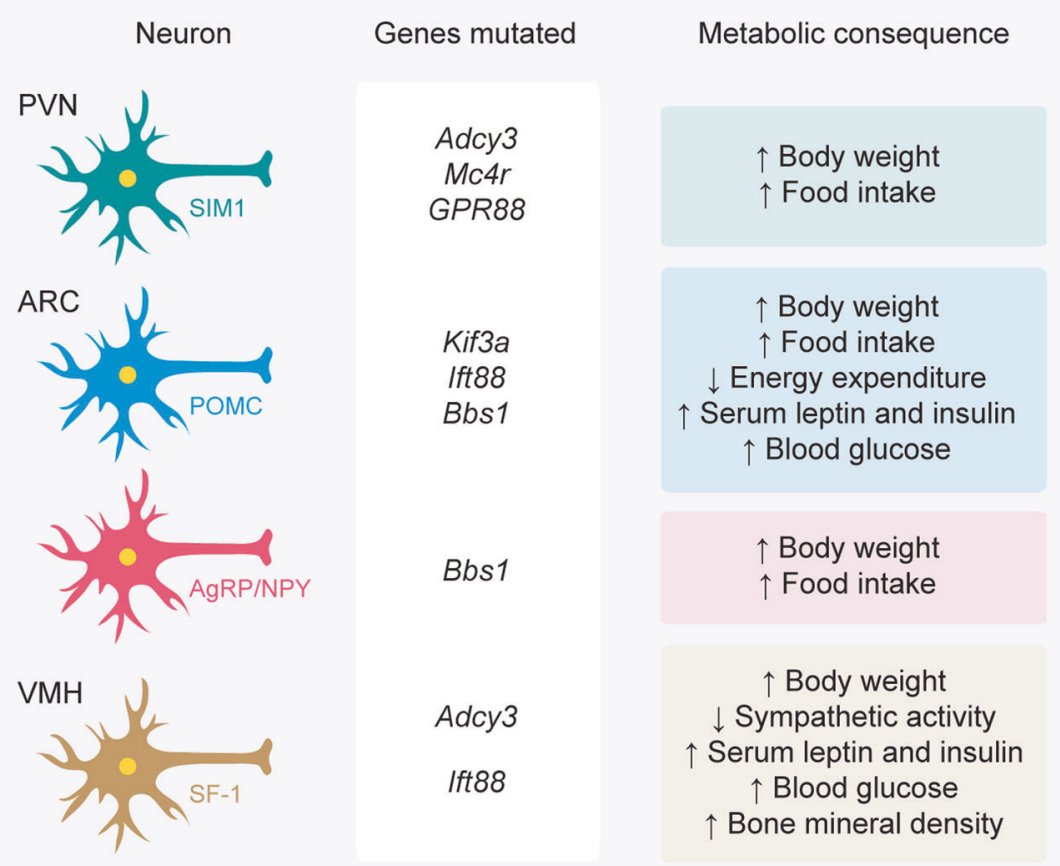

Fig. 3 Ciliary genes in the hypothalamic nuclei involved in metabolic dysfunction. Simplified overview of metabolic changes as a consequence of the mutation of ciliary genes in the indicated hypothalamic neurons. Notably, primary cilia play a distinct homeostatic role in each hypothalamic nucleus. 
portrayed as significantly increased rebound food intake, as well as the effects of an increase in energy expenditure, were blunted, indicating that the deletion of primary cilia in the VMH blunted leptin sensitivity [41].

Taken together, the current data imply that the impact of primary cilia on leptin action may differ among neuron types. To rationalize the molecular mechanism underlying between primary cilia and leptin in energy homeostasis, further observations should be considered to determine (i) whether the leptin receptor exists in the primary cilium and (ii) distinct neuronal populations linking leptin action and the role of primary cilia.

\section{CONCLUSIONS}

The regulation of energy balance is complex and governed by diverse neuronal factors. Several recent studies have revealed the distinct roles of hypothalamic primary cilia in controlling energy balance (Fig. 3) [41, 61, 62, 64, 77]. Although the functional importance of cilia has been determined, studies are needed to further reveal and to gain full understanding of the molecular composition of neuronal cilia and their precise roles in modulating energy homeostasis, primarily by determining the distinct roles that hypothalamic primary cilia play in different neurons. Moreover, given that cilia biogenic genes such as Ift88 have ciliaindependent roles in tissue development, direct evidence that defects in ciliary formation per se are necessary for acquisition of a pathological phenotype is still needed. Additionally, since primary cilia have been known to contribute to neural development, it would be reasonable to examine whether neuronal cilia influence the formation of the neuronal circuit [64, 83, 84]. In summary, studies of hypothalamic primary cilia offer great potential to gain understanding of other aspects of energy homeostasis regulation through the central nervous system and possibly provide a new strategy to overcome metabolic disorders.

\section{REFERENCES}

1. Marshall, W. F. \& Nonaka, S. Cilia: tuning in to the cell's antenna. Curr. Biol. 16, R604-R614 (2006).

2. Ishikawa, H. \& Marshall, W. F. Ciliogenesis: building the cell's antenna. Nat. Rev. Mol. Cell. Biol. 12, 222-234 (2011).

3. Pedersen, L. B. \& Rosenbaum, J. L. Intraflagellar transport (IFT) role in ciliary assembly, resorption and signalling. Curr. Top. Dev. Biol. 85, 23-61 (2008).

4. Singla, V. \& Reiter, J. F. The primary cilium as the cell's antenna: signaling at a sensory organelle. Science 313, 629-633 (2006).

5. Pazour, G. J. et al. Chlamydomonas IFT88 and its mouse homologue, polycystic kidney disease gene $\operatorname{tg} 737$, are required for assembly of cilia and flagella. J. Cell Biol. 151, 709-718 (2000).

6. Hildebrandt, F., Benzing, T., \& Katsanis, N. Ciliopathies. N. Engl. J. Med. 364, 1533-1543 (2011).

7. Ansley, S. J. et al. Basal body dysfunction is a likely cause of pleiotropic BardetBiedl syndrome. Nature 425, 628-633 (2003).

8. Tobin, J. L. \& Beales, P. L. The nonmotile ciliopathies. Genet. Med. 11, 386-402 (2009).

9. Davenport, J. R. et al. Disruption of intraflagellar transport in adult mice leads to obesity and slow-onset cystic kidney disease. Curr. Biol. 17, 1586-1594 (2007).

10. Engle, S.E., Bansal, R., Antonellis, P.J. \& Berbari, N.F. Cilia signaling and obesity. Semin. Cell. Dev. Biol. 110, 43-50 (2021).

11. Satir, P. CILIA: before and after. Cilia 6, 1 (2017).

12. Song, D. K., Choi, J. H. \& Kim, M. S. Primary cilia as a signaling platform for control of energy metabolism. Diabetes Metab. J. 42, 117-127 (2018).

13. Lechan, R.M. \& Toni, R. Functional anatomy of the hypothalamus and pituitary. In Endotext (eds. Feingold, K.R. et al.) (MDText.com, Inc., 2000).

14. Anand, B. K. \& Brobeck, J. R. Hypothalamic control of food intake in rats and cats. Yale J. Biol. Med. 24, 123-140 (1951).

15. Rui, L. Brain regulation of energy balance and body weight. Rev. Endocr. Metab. Disord. 14, 387-407 (2013).

16. Sainsbury, A. \& Zhang, L. Role of the arcuate nucleus of the hypothalamus in regulation of body weight during energy deficit. Mol. Cell Endocrinol. 316, 109-119 (2010).
17. Simpson, K. A., Martin, N. M. \& Bloom, S. R. Hypothalamic regulation of food intake and clinical therapeutic applications. Arq. Bras. Endocrinol. Metab. 53, 120-128 (2009).

18. Belgardt, B. F. \& Bruning, J. C. CNS leptin and insulin action in the control of energy homeostasis. Ann. NY Acad. Sci. 1212, 97-113 (2010).

19. Suyama, S. et al. Glucose level determines excitatory or inhibitory effects of adiponectin on arcuate POMC neuron activity and feeding. Sci. Rep. 6, 30796 (2016).

20. Waterson, M. J. \& Horvath, T. L. Neuronal regulation of energy homeostasis: beyond the hypothalamus and feeding. Cell Metab. 22, 962-970 (2015).

21. Baskin, D. G., Hahn, T. M. \& Schwartz, M. W. Leptin sensitive neurons in the hypothalamus. Horm. Metab. Res. 31, 345-350 (1999).

22. Kwon, O., Kim, K. W. \& Kim, M. S. Leptin signalling pathways in hypothalamic neurons. Cell Mol. Life Sci. 73, 1457-1477 (2016).

23. Williams, G. et al. The hypothalamus and the control of energy homeostasis: different circuits, different purposes. Physiol. Behav. 74, 683-701 (2001).

24. Cone, R. D. Anatomy and regulation of the central melanocortin system. Nat. Neurosci. 8, 571-578 (2005).

25. Atasoy, D., Betley, J. N., Su, H. H. \& Sternson, S. M. Deconstruction of a neural circuit for hunger. Nature 488, 172-177 (2012).

26. Garfield, A. S. et al. A neural basis for melanocortin-4 receptor-regulated appetite. Nat. Neurosci. 18, 863-871 (2015).

27. Li, M. M. et al. The paraventricular hypothalamus regulates satiety and prevents obesity via two genetically distinct circuits. Neuron 102, 653-667.e6 (2019)

28. Shah, B. P. et al. MC4R-expressing glutamatergic neurons in the paraventricular hypothalamus regulate feeding and are synaptically connected to the parabrachial nucleus. Proc. Natl Acad. Sci. USA 111, 13193-13198 (2014).

29. Todd, W. D. et al. A hypothalamic circuit for the circadian control of aggression. Nat. Neurosci. 21, 717-724 (2018).

30. Leibowitz, S. F., Hammer, N. J. \& Chang, K. Hypothalamic paraventricular nucleus lesions produce overeating and obesity in the rat. Physiol. Behav. 27, 1031-1040 (1981).

31. Sims, J. S. \& Lorden, J. F. Effect of paraventricular nucleus lesions on body weight, food intake and insulin levels. Behav. Brain Res. 22, 265-281 (1986).

32. Rothwell, N. J. Central effects of CRF on metabolism and energy balance. Neurosci. Biobehav. Rev. 14, 263-271 (1990).

33. Lechan, R. M. \& Fekete, C. The TRH neuron: a hypothalamic integrator of energy metabolism. Prog. Brain Res. 153, 209-235 (2006).

34. Hetherington, A. W. \& Ranson, S. W. Hypothalamic lesions and adiposity in the rat. Anat. Rec. 78, 149-172 (1940).

35. Shimazu, T. Central nervous system regulation of liver and adipose tissue metabolism. Diabetologia 20, 343-356 (1981).

36. Dhillon, $\mathrm{H}$. et al. Leptin directly activates SF1 neurons in the $\mathrm{VMH}$, and this action by leptin is required for normal body-weight homeostasis. Neuron 49, 191-203 (2006).

37. Kim, K. W. et al. Steroidogenic factor 1 directs programs regulating diet-induced thermogenesis and leptin action in the ventral medial hypothalamic nucleus. Proc. Natl Acad. Sci. USA 108, 10673-10678 (2011).

38. Fujikawa, T. et al. P110beta in the ventromedial hypothalamus regulates glucose and energy metabolism. Exp. Mol. Med. 51, 52-59 (2019).

39. Kim, K. W. et al. FOXO1 in the ventromedial hypothalamus regulates energy balance. J. Clin. Invest. 122, 2578-2589 (2012).

40. Minokoshi, Y., Saito, M. \& Shimazu, T. Sympathetic denervation impairs responses of brown adipose tissue to VMH stimulation. Am. J. Physiol. 251, R1005-R1008 (1986).

41. Sun, J.S. et al. Ventromedial hypothalamic primary cilia control energy and skeletal homeostasis.J. Clin. Invest. 131, e138107 (2021).

42. Zhao, L. et al. Central nervous system-specific knockout of steroidogenic factor 1 results in increased anxiety-like behavior. Mol. Endocrinol. 22, 1403-1415 (2008).

43. Kim, K. W., Zhao, L. \& Parker, K. L. Central nervous system-specific knockout of steroidogenic factor 1. Mol. Cell Endocrinol. 300, 132-136 (2009).

44. Ball, G. G. Self-stimulation in the ventromedial hypothalamus. Science 178, 72-73 (1972).

45. Takahashi, A., Ishimaru, H., Ikarashi, Y., Kishi, E. \& Maruyama, Y. Effects of ventromedial hypothalamus stimulation on glycogenolysis in rat liver using in vivo microdialysis. Metabolism 46, 897-901 (1997).

46. Sakaguchi, T., Arase, K. \& Bray, G. A. Sympathetic activity and food intake of rats with ventromedial hypothalamic lesions. Int J. Obes. 12, 285-291 (1988).

47. Vander Tuig, J. G., Knehans, A. W. \& Romsos, D. R. Reduced sympathetic nervous system activity in rats with ventromedial hypothalamic lesions. Life Sci. 30, 913-920 (1982).

48. $\mathrm{Xu}, \mathrm{B}$. et al. Brain-derived neurotrophic factor regulates energy balance downstream of melanocortin-4 receptor. Nat. Neurosci. 6, 736-742 (2003). 
49. Sternson, S. M., Shepherd, G. M. \& Friedman, J. M. Topographic mapping of VMH $\rightarrow$ arcuate nucleus microcircuits and their reorganization by fasting. Nat. Neurosci. 8, 1356-1363 (2005).

50. Stuber, G. D. \& Wise, R. A. Lateral hypothalamic circuits for feeding and reward. Nat. Neurosci. 19, 198-205 (2016).

51. Qualls-Creekmore, E. \& Munzberg, H. Modulation of feeding and associated behaviors by lateral hypothalamic circuits. Endocrinology 159, 3631-3642 (2018).

52. Bernardis, L. L. \& Bellinger, L. L. The lateral hypothalamic area revisited: ingestive behavior. Neurosci. Biobehav. Rev. 20, 189-287 (1996).

53. Qiu, L., LeBel, R. P., Storm, D. R. \& Chen, X. Type 3 adenylyl cyclase: a key enzyme mediating the CAMP signaling in neuronal cilia. Int J. Physiol. Pathophysiol. Pharmacol. 8, 95-108 (2016).

54. Steegborn, C. Structure, mechanism, and regulation of soluble adenylyl cyclases similarities and differences to transmembrane adenylyl cyclases. Biochim. Biophys. Acta 1842, 2535-2547 (2014).

55. Glantz, S. B., Amat, J. A. \& Rubin, C. S. cAMP signaling in neurons: patterns of neuronal expression and intracellular localization for a novel protein, AKAP 150, that anchors the regulatory subunit of CAMP-dependent protein kinase II beta. Mol. Biol. Cell 3, 1215-1228 (1992).

56. Bishop, G. A., Berbari, N. F., Lewis, J. \& Mykytyn, K. Type III adenylyl cyclase localizes to primary cilia throughout the adult mouse brain. J. Comp. Neurol. 505, 562-571 (2007).

57. Wang, Z. et al. Adult type 3 adenylyl cyclase-deficient mice are obese. PLOS ONE 4, e6979 (2009).

58. Schou, K. B., Pedersen, L. B. \& Christensen, S. T. Ins and outs of GPCR signaling in primary cilia. EMBO Rep. 16, 1099-1113 (2015).

59. Lee, H., Song, J., Jung, J. H. \& Ko, H. W. Primary cilia in energy balance signaling and metabolic disorder. BMB Rep. 48, 647-654 (2015).

60. Oh, E. C., Vasanth, S. \& Katsanis, N. Metabolic regulation and energy homeostasis through the primary Cilium. Cell Metab. 21, 21-31 (2015).

61. Seo, S. et al. Requirement of Bardet-Biedl syndrome proteins for leptin receptor signaling. Hum. Mol. Genet. 18, 1323-1331 (2009).

62. Guo, D. F. et al. The BBSome controls energy homeostasis by mediating the transport of the leptin receptor to the plasma membrane. PLoS Genet. 12, e1005890 (2016)

63. Han, Y. M. et al. Leptin-promoted cilia assembly is critical for normal energy balance. J. Clin. Invest. 124, 2193-2197 (2014).

64. Lee, C. H. et al. Primary cilia mediate early life programming of adiposity through lysosomal regulation in the developing mouse hypothalamus. Nat. Commun. 11, 5772 (2020).

65. Lechtreck, K. F. et al. The Chlamydomonas reinhardtii BBSome is an IFT cargo required for export of specific signaling proteins from flagella. J. Cell Biol. 187, 1117-1132 (2009).

66. Nachury, M. V. et al. A core complex of BBS proteins cooperates with the GTPase Rab8 to promote ciliary membrane biogenesis. Cell 129, 1201-1213 (2007).

67. Oh, E. C. \& Katsanis, N. Cilia in vertebrate development and disease. Development 139, 443-448 (2012).

68. Berbari, N. F., Lewis, J. S., Bishop, G. A., Askwith, C. C. \& Mykytyn, K. Bardet-Bied syndrome proteins are required for the localization of $G$ protein-coupled receptors to primary cilia. Proc. Natl Acad. Sci. USA 105, 4242-4246 (2008).

69. Rahmouni, K. et al. Leptin resistance contributes to obesity and hypertension in mouse models of Bardet-Biedl syndrome. J. Clin. Invest. 118, 1458-1467 (2008).

70. Guo, D. F. et al. The BBSome in POMC and AgRP neurons is necessary for body weight regulation and sorting of metabolic receptors. Diabetes $\mathbf{6 8}, 1591-1603$ (2019).

71. Morton, G. J., Meek, T. H. \& Schwartz, M. W. Neurobiology of food intake in health and disease. Nat. Rev. Neurosci. 15, 367-378 (2014).

72. Huszar, D. et al. Targeted disruption of the melanocortin-4 receptor results in obesity in mice. Cell 88, 131-141 (1997).

73. Vaisse, C. et al. Melanocortin-4 receptor mutations are a frequent and heterogeneous cause of morbid obesity. J. Clin. Invest. 106, 253-262 (2000).

74. Vaisse, C., Clement, K., Guy-Grand, B. \& Froguel, P. A frameshift mutation in human MC4R is associated with a dominant form of obesity. Nat. Genet. 20, 113-114 (1998).
75. Siljee, J. E. et al. Subcellular localization of MC4R with ADCY3 at neuronal primary cilia underlies a common pathway for genetic predisposition to obesity. Nat. Genet. 50, 180-185 (2018).

76. Nordman, S. et al. Genetic variation of the adenylyl cyclase 3 (AC3) locus and its influence on type 2 diabetes and obesity susceptibility in Swedish men. Int $\mathrm{J}$. Obes. (Lond.) 32, 407-412 (2008).

77. Cao, H., Chen, X., Yang, Y. \& Storm, D. R. Disruption of type 3 adenylyl cyclase expression in the hypothalamus leads to obesity. Integr. Obes. Diabetes 2, 225-228 (2016).

78. Allison, M. B. \& Myers, M. G. Jr 20 years of leptin: connecting leptin signaling to biological function. J. Endocrinol. 223, T25-T35 (2014).

79. Friedman, J. M. \& Mantzoros, C. S. 20 years of leptin: from the discovery of the leptin gene to leptin in our therapeutic armamentarium. Metabolism 64, 1-4 (2015).

80. Myers, M. G. Jr, Leibel, R. L., Seeley, R. J. \& Schwartz, M. W. Obesity and leptin resistance: distinguishing cause from effect. Trends Endocrinol. Metab. 21, 643-651 (2010).

81. Porte, D. et al. Obesity, diabetes and the central nervous system. Diabetologia 41, 863-881 (1998).

82. Berbari, N. F. et al. Leptin resistance is a secondary consequence of the obesity in ciliopathy mutant mice. Proc. Natl Acad. Sci. USA 110, 7796-7801 (2013).

83. Park, S. M., Jang, H. J. \& Lee, J. H. Roles of primary cilia in the developing brain. Front. Cell. Neurosci. 13, 218 (2019).

84. Guadiana, S. M. et al. Arborization of dendrites by developing neocortical neurons is dependent on primary cilia and type 3 adenylyl cyclase. J. Neurosci. 33, 2626-2638 (2013).

\section{ACKNOWLEDGEMENTS}

This work was supported by the National Research Foundation, Korea (2016R1A5A2008630 and 2021R1A2C4002011), and Yonsei University College of Dentistry (6-2019-0019) for K.W.K.

\section{COMPETING INTERESTS}

The authors declare no competing interests.

\section{ADDITIONAL INFORMATION}

Correspondence and requests for materials should be addressed to K.W.K.

Reprints and permission information is available at http://www.nature.com/ reprints

Publisher's note Springer Nature remains neutral with regard to jurisdictional claims in published maps and institutional affiliations.

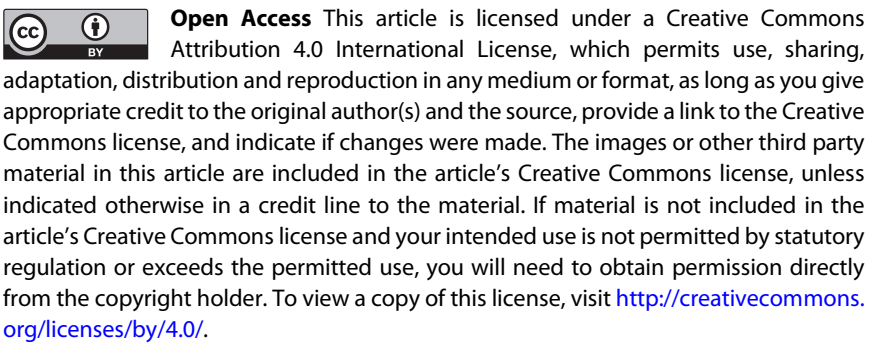

(c) The Author(s) 2021 